\title{
What is the Correct Definition of Average Pressure?
}

\author{
S. Korteland - S. Bottero - S. M. Hassanizadeh • \\ C. W. J. Berentsen
}

Received: 2 January 2008 / Accepted: 14 October 2009 / Published online: 7 November 2009

(C) The Author(s) 2009. This article is published with open access at Springerlink.com

\begin{abstract}
Use of a correct definition of average pressure is important in numerical modeling of oil reservoirs and aquifers, where the simulated domain can be very large. Also, the average pressure needs to be defined in the application of pore-network modeling of (two-phase) flow in porous media, as well as in the (theoretical) upscaling of flow equations. Almost always the so-called intrinsic phase-volume average operator, which weighs point pressure values with point saturation values, is employed. Here, we introduce and investigate four other potentially plausible averaging operators. Among them is the centroid-corrected phase-average pressure, which corrects the intrinsic phase-volume average pressure for the distance between the centroid of the averaging volume and the phase. We consider static equilibrium of two immiscible fluids in a homogeneous, one-dimensional, vertical porous medium domain under a series of (static) drainage conditions. An important feature of static equilibrium is that the total potential (i.e., the sum of pressure and gravity potentials) is constant for each phase over the whole domain. Therefore, its average will be equal to the same constant. It is argued that the correct average pressure must preserve the fact that fluid potentials are constant. We have found that the intrinsic phase-volume average pressure results in a gradient in the total phase potential, i.e., the above criterion is violated. In fact, only the centroid-corrected operator satisfies this criterion. However, at high saturations, use of the centroid-corrected average can give rise to negative values of the difference between the average nonwetting and wetting phase pressures. For main drainage, differences among
\end{abstract}

\section{S. Korteland}

Department of Geotechnology, Delft University of Technology, P.O. Box 5048, 2600 GA, Delft, The Netherlands

S. Bottero · S. M. Hassanizadeh $(\varangle) \cdot$ C. W. J. Berentsen

Department of Earth Sciences, Utrecht University, P.O.Box 80021, 3508 TA,

Utrecht, The Netherlands

e-mail: Hassanizadeh@geo.uu.nl

Present Address:

C. W. J. Berentsen

Department of Geotechnology, Delft University of Technology, P.O. Box 5048,

2600 GA, Delft, The Netherlands 
various averaging operators are significantly less because both phases are present initially, such that the difference between the centroids of phases, and the middle of the domain are relatively small.

Keywords Porous media - Volume averaging - Macroscale pressure - Average pressure $\cdot$ Capillary pressure

\section{List of Symbols}

Latin Symbols

$\hat{\boldsymbol{e}}_{f} \quad$ Unit vector in the direction of flow [-]

$g \quad$ Gravitational acceleration $\left[\mathrm{LT}^{-2}\right]$

$H \quad$ Height of the domain [L]

$P_{\alpha} \quad$ Pressure in phase $\alpha\left[\mathrm{ML}^{-1} \mathrm{~T}^{-2}\right]$

$P_{\mathrm{c}} \quad$ Capillary pressure $\left[\mathrm{ML}^{-1} \mathrm{~T}^{-2}\right]$

$P_{\mathrm{d}} \quad$ Entry pressure $\left[\mathrm{ML}^{-1} \mathrm{~T}^{-2}\right]$

$\Delta P$ nonwetting phase bottom boundary overpressure $\left[\mathrm{ML}^{-1} \mathrm{~T}^{-2}\right]$

$S_{\alpha} \quad$ Saturation for phase $\alpha[-]$

$S_{\mathrm{e}} \quad$ Effective saturation [-]

$S_{\mathrm{wr}} \quad$ Residual wetting phase saturation [-]

$S_{\mathrm{nr}} \quad$ Residual nonwetting phase saturation [-]

$V \quad$ Volume of integration $\left[\mathrm{L}^{3}\right]$

$V_{\alpha} \quad$ Volume of integration of phase $\alpha\left[\mathrm{L}^{3}\right]$

$z \quad$ Position vector [L]

$\langle z\rangle \quad$ Centroid of averaging volume [L]

$z_{f} \quad$ Position of the infiltration front in the domain [-]

\section{Greek Symbols}

$\eta_{\alpha} \quad$ Indicator function for phase $\alpha$ [-]

$\lambda$ Brooks-Corey pore-size distribution parameter [-]

$\rho_{\alpha} \quad$ Density of phase $\alpha\left[\mathrm{ML}^{-3}\right]$

$\rho \quad$ Density ratio [-]

$\Phi_{\alpha} \quad$ Potential of phase $\alpha\left[\mathrm{ML}^{-1} \mathrm{~T}^{-2}\right]$

\section{Special Notations}

\langle\rangle$\quad$ Potential-based average operator

\langle\rangle$^{i} \quad$ Intrinsic phase-volume average operator

\langle\rangle$^{s p} \quad$ Simple phase-average operator

[]$_{1}$ Centroid-corrected phase-average operator

\langle\rangle$^{s} \quad$ Simple average operator

... (prime sign) Dimensionless form of variable [ ]

\section{Subscripts}

$\alpha$ Phase, either wetting (w) or nonwetting (n)

$\mathrm{n}$ nonwetting phase

w Wetting phase 


\section{Introduction}

When numerical models are used to investigate flow processes in reservoirs or aquifers, the simulated domain commonly has a size on the scale of hundreds of meters to kilometers. However, oil reservoirs and aquifers usually have complex structures, consisting of heterogeneities on different length scales. In an ideal situation, the numerical flow simulator should include all these variations. Unfortunately, the current computational power and our ability to characterize the subsurface are not sufficient to include such detailed structures. As a result, the numerical grid size is often much larger than the scale of medium heterogeneities. For instance, for a medium-sized oil reservoir, the grid in a numerical simulator may have a resolution on the decameter scale, whereas heterogeneities may exist at the scale of centimeters. For such grid blocks, average parameter values are needed. These upscaled parameters should ideally be derived from the fine-scale parameters inside the grid blocks. This requires appropriate definitions of average values for various properties. Also, in pore-network modeling of (two-phase) flow in porous media, average values need to be specified. Moreover, in theoretical upscaling of (two-phase) flow, starting from Navier-Stokes equation or Darcy-flow equation, average properties need to be defined.

One of the most important variables is fluid pressure. We submit that it is not clear, yet, what the correct definition of average pressure should be. Recently, there has been some discussion in the literature in this regard (Gray and Miller 2004, 2007; Nordbotten et al. 2007, 2008).

In the vast literature on porous medium averaging from pore to Darcy scale, almost invariably the intrinsic phase-volume average pressure is employed for defining the macroscale pressure (see e.g., Whitaker 1997; Gray 1975; Gray and O'Neill 1976; Neumann 1977; Quintard and Whitaker 1994a,b; Bear 1988; Bachmat and Bear 1986; Bear and Bachmat 1986; Gray and Miller 2004):

$$
\left\langle P_{\alpha}\right\rangle^{i}=\frac{1}{V_{\alpha}} \int_{V_{\alpha}} P_{\alpha} \mathrm{d} V,
$$

where $P_{\alpha}$ is the pressure of phase $\alpha$, and $V_{\alpha}$ denotes the domain (and the volume) occupied by the $\alpha$-phase. It is almost always (implicitly) suggested that the intrinsic phase-volume average pressure corresponds to the pressure that we actually measure (see e.g., Gray and Miller 2007). To the best of our knowledge, however, this presumption has never been proven by means of measurements. Ideally, such an experiment would constitute measuring pressure inside pores of a given volume by a small-scale sensor and measuring the macroscale pressure of the whole volume by a large-scale sensor. Although such an experiment has not been carried out yet, we can identify realistic situations, where pressure obtained from a certain way of measurement will not be equal to the intrinsic phase-volume average pressure.

Consider the simple case of hydrostatic equilibrium in a saturated soil column as depicted in Fig. 1a. The pressure at a given elevation $z_{1}$ can be measured by a manometer. The pressure at this elevation is equal to $\rho_{\mathrm{w}} g h_{1}$, where $\rho_{\mathrm{w}}$ is mass density of water and $h_{1}$ is the height of water column above that elevation. Obviously, this is the average pressure over the opening of the manometer, centered at $z_{1}$. It is also equal to the point pressure at the centroid of the opening $z_{1}$ (see Fig. 1b). Now, assume that a closer examination of the porous medium shows that the pore sizes are not uniformly distributed over the manometer opening but, as shown schematically in Fig. 1c, there are larger pores in the lower half of the opening. This means that, due to the hydrostatic gradient in pressure, the intrinsic phase-volume average pressure will be larger than $\rho_{\mathrm{w}} g h_{1}$, which is the pressure measured by the manometer. The 
(a)

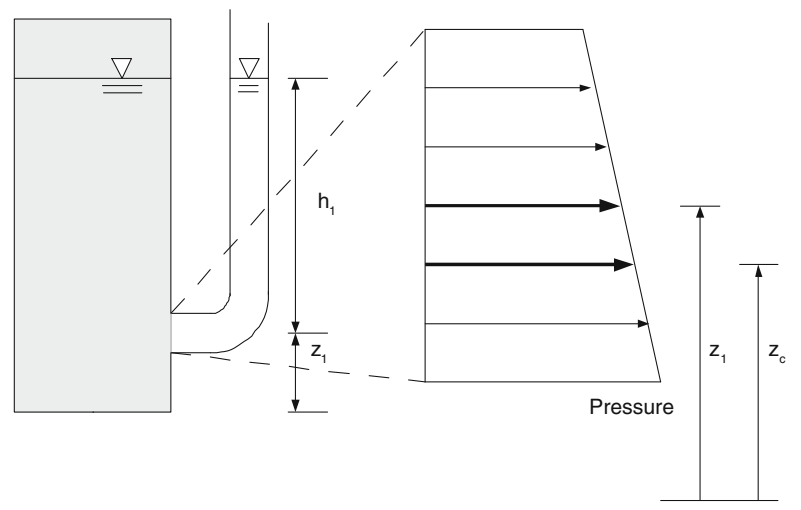

(c)

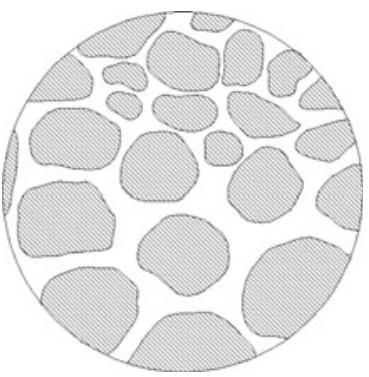

Fig. 1 a Schematic representation of manometer used to measure pressure at a given elevation $\mathrm{z}_{1}$ in a porous medium column fully saturated with water (drawing is not to scale). b Pressure distribution across the manometer opening. $\mathbf{c}$ Schematic representation of pore distribution at the manometer opening, showing larger pores in the lower half of the opening

reason for this discrepancy is that the centroid of the water phase (in this case, the pore space), denoted by $z_{\mathrm{c}}$, does not coincide with the centroid of the averaging volume (i.e., the manometer opening). In fact, the intrinsic phase-volume average pressure will be equal to the point pressure at $z_{\mathrm{c}}$, the centroid of the pore space. A similar discussion on this issue can be found in Nordbotten et al. (2007), who averaged the microscale flow equation for cases with a porosity gradient. They showed that the classical definition of average pressure (the intrinsic phase-volume average) does not lead to the classical Darcy's law, but gives rise to a nonphysical gravity term. Due to the gradient in porosity, there is a systematic lengthscale-dependent difference between the centroid of the phase and the averaging volume. Nordbotten et al. (2007) proposed a new definition for average pressure, which was based on a Taylor expansion around the intrinsic phase-volume average of the microscopic pressure.

Gray and Miller (2004) also considered single-phase flow in a domain with a macroscale gradient in porosity. They averaged Darcy scale flow equations and showed that intrinsic phase-volume average pressure incorrectly prescribes a flow that does not actually exist. Their conclusion was that one has to work with average hydraulic head (pressure head + gravity head) instead of average pressure. However, this is not feasible in the case of two-phase flow because we need to work with fluid pressures to be able to define capillary pressure.

Now, consider averaging pressure in two-phase flow problems. Even when the pore space in an averaging volume is uniformly distributed, it is usually not uniformly occupied by the two phases. Therefore, in most cases, the centroids of the two phases do not coincide with the centroid of the averaging volume. In such cases, it is not clear that the intrinsic phasevolume average pressure corresponds to the measured macroscale pressure. In a second paper, Nordbotten et al. (2008) extended their new definition of pressure to multi-phase flow in porous media. As these authors note, even in the case of homogeneous porous media, the intrinsic phase-volume average pressure leads to additional terms in Darcy's law because, in general, there will be gradients in saturation and pressure on all scales. The new averaging operator defined by Nordbotten et al. (2008), to which we refer as the centroid-corrected average, is one of the averaging operators investigated in this article. In fact, the utility of that averaging operator and how it compares to other averaging operators is not investigated yet. 
In particular, the effect of choice of averaging operator on the upscaling of capillary pressure is not studied at all.

Despite the problems with the definition of average pressure outlined above, there is not much discussion in the literature. In this article, the upscaling of fluid pressure from the Darcy scale to larger scales is investigated for a column containing two immiscible fluids. Four different averaging operators are used to obtain average pressure. The consequences of the choice of averaging pressure operator for the capillary pressure-saturation relationship will be studied for situations representing static drainage experiments. We also discuss situations, where the choice of averaging pressure operator may not be an important issue. Averaging operators are introduced in Sect. 2. Section 3 contains a description of the model used. In Sect. 4, results are discussed. Section 5 contains a summary and discussion.

\section{Averaging Operators}

Consider a porous medium for which the porosity and capillary pressure-saturation relationship are known. Without loss of generality, we assume that these properties do not change with time. Moreover, we assume that porosity is constant in space. For given boundary conditions, one can derive saturation and fluid pressure distributions under static conditions. Thus, we assume that saturations $S_{\alpha}$ and pressures $P_{\alpha}$ are known functions of time and space.

Traditionally, the macroscale pressure is defined as the so-called intrinsic phase-volume average pressure (Eq. 1.1). For two-phase flow in a porous medium with constant porosity, this averaging operator becomes:

$$
\left\langle P_{\alpha}\right\rangle^{i}=\frac{1}{\int_{V} S_{\alpha} \mathrm{d} V} \int_{V} S_{\alpha} P_{\alpha} \mathrm{d} V \quad \alpha=\mathrm{w}, \mathrm{n},
$$

where $S_{\alpha}$ is the saturation of phase $\alpha$. Phase $\alpha$ is either wetting (w) or nonwetting (n). Although the integral limit indicates that the averaging is carried out over the whole domain, in fact, the averaging is performed only over that part of the domain where the $\alpha$-phase is actually present $\left(S_{\alpha}>0\right)$. In early stages of a front displacement, this could be only a small part of the whole domain for the nonwetting phase. This averaging operator is analogous to the intrinsic phase-volume average operator used in upscaling from pore scale to Darcy scale (see e.g., Gray 1975; Whitaker 1997; Hassanizadeh and Gray 1979). In addition, this averaging operator has been used for averaging from Darcy scale to higher scales (e.g., AtaieAshtiani et al. 2001, 2002; Das et al. 2004; Manthey et al. 2005), and in pore-network models, for determining macroscale pressure fields (e.g., Dahle and Celia 1999; Gielen et al. 2004, 2005; Dahle et al. 2005; Gielen 2007).

As given in Sect. 1, the average pressures defined by Eq. 2.1 are actually not calculated over the same domain whenever a front exists. Also, in general, the centroids of the two phases do not necessarily coincide with each other or with the centroid of the sample. In order to partially alleviate this problem, we may choose to define an average pressure without weighing it with the phase volumes:

$$
\left\langle P_{\alpha}\right\rangle^{s p}=\frac{1}{\int_{V} \eta_{\alpha} \mathrm{d} V} \int_{V} P_{\alpha} \eta_{\alpha} \mathrm{d} V \quad \alpha=\mathrm{w}, \mathrm{n},
$$

where $\eta_{\alpha}$ represents an indicator function, defined as: 


$$
\eta_{\alpha}=\left\{\begin{array}{lll}
1 & \text { if } & S_{\alpha}>0 \\
0 & \text { if } & S_{\alpha}=0
\end{array}\right\}
$$

The indicator function ensures that averaging is performed over regions where the $\alpha$-phase is actually present. We shall refer to $\left\langle P_{\alpha}\right\rangle^{s p}$ as the simple phase-average pressure. While this average does not weigh pressure by the phase volume (i.e., saturation), it is still defined at the centroid of the actual domain occupied by a phase, and not at the centroid of the averaging volume. Therefore, we consider yet another definition, for which averaging is carried out over the whole domain:

$$
\left\langle P_{\alpha}\right\rangle^{s}=\frac{1}{\int_{V} \mathrm{~d} V} \int_{V} P_{\alpha} \mathrm{d} V \quad \alpha=\mathrm{w}, \mathrm{n} .
$$

Here, it is assumed that $P_{\alpha}$ is defined everywhere in the domain of interest even if the $\alpha$-phase is not present everywhere. In the definition above, the averaging domain is exactly the same for both the nonwetting and the wetting phase. This average pressure will be referred to in this article as the simple average pressure.

As given in Sect.1, a new definition of macroscale quantities has been proposed by Nordbotten et al. 2008. In fact, these authors introduce a family of macroscale pressures $\left[P_{\alpha}\right]_{n}$, where $n$ refers to the order of the approximation to a smooth macroscale function $\left[P_{\alpha}\right]$. Nordbotten et al. 2008 obtain an expression for macroscale pressure from its microscale equivalent. However, their approach and definition can also be used for upscaling from core scale to higher scales.

We propose to use the first-order approximation of the macroscale pressure, given by:

$$
\left[P_{\alpha}\right]_{1}=\left\langle P_{\alpha}\right\rangle^{i}+\frac{1}{\nabla \cdot\left\langle z_{\alpha}\right\rangle^{i}}\left(\langle z\rangle-\left\langle z_{\alpha}\right\rangle^{i}\right) \cdot \nabla\left\langle P_{\alpha}\right\rangle^{i} \quad \alpha=\mathrm{w}, \mathrm{n}
$$

where $\left\langle P_{\alpha}\right\rangle^{i}$ is the intrinsic phase-volume average pressure of phase $\alpha$ defined by Eq. 2.1, $\langle z\rangle$ is the position to which $\left[P_{\alpha}\right]_{1}$ is assigned (usually the centroid of the sample REV), and $\left\langle z_{\alpha}\right\rangle^{i}$ is the intrinsic phase-volume average of position vector $z$. Equation 2.5 suggests that the first-order approximation of the macroscale pressure is equal to the intrinsic phasevolume average pressure $\left\langle P_{\alpha}\right\rangle^{i}$, corrected for the distance between the centroid of the averaging volume $(\langle z\rangle)$, and the centroid of the phase $\left(\left\langle z_{\alpha}\right\rangle^{i}\right)$. We refer to this macroscale pressure as the centroid-corrected phase-average pressure.

In Eq. 2.5, derivatives of the intrinsic phase-average pressure $\left\langle P_{\alpha}\right\rangle^{i}$ and position vector $\left\langle z_{\alpha}\right\rangle^{i}$ are needed. These can be calculated for the particular situation of a vertical domain with flow from bottom to top as follows (see the Appendix for the derivation of these equations):

$$
\begin{aligned}
\frac{\partial}{\partial z}\left\langle P_{\alpha}\right\rangle^{i} & \equiv \frac{1}{\left\langle S_{\alpha}\right\rangle H}\left[-\left\langle P_{\alpha}\right\rangle^{i}\left(S_{\alpha}^{\text {top }}-S_{\alpha}^{\text {bottom }}\right)+\left(S_{\alpha}^{\text {top }} P_{\alpha}^{\text {top }}-S_{\alpha}^{\text {bottom }} P_{\alpha}^{\text {bottom }}\right)\right] \\
\frac{\partial}{\partial z}\left\langle z_{\alpha}\right\rangle^{i} & \equiv \frac{1}{\left\langle S_{\alpha}\right\rangle H}\left[-\left\langle z_{\alpha}\right\rangle^{i}\left(S_{\alpha}^{\text {top }}-S_{\alpha}^{\text {bottom }}\right)+\left(S_{\alpha}^{\text {top }} z^{\text {top }}-S_{\alpha}^{\text {bottom }} z^{\text {bottom }}\right)\right],
\end{aligned}
$$

where $H$ denotes the length of the domain. The superscripts "top" and "bottom" refer to the value of the variable at top and bottom of the domain, respectively. 
(a)

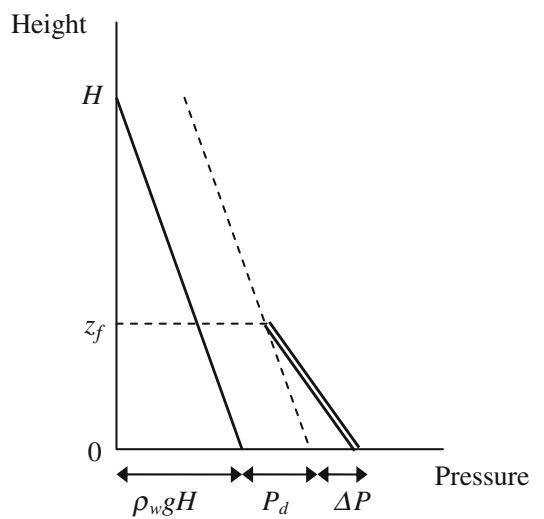

(b)

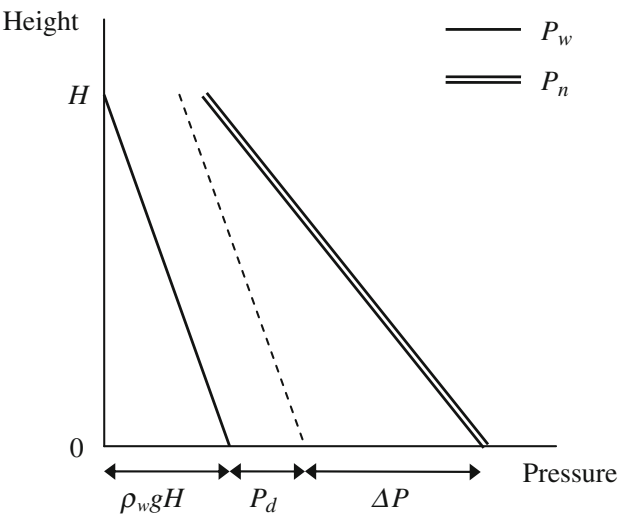

Fig. 2 Schematic pressure distribution for the wetting and nonwetting phase in a vertical one-dimensional homogeneous domain at static equilibrium. The nonwetting phase has a higher density than the wetting phase, and infiltrates the domain from below. a The nonwetting phase has infiltrated the domain up to $z_{f}$. b The nonwetting phase has infiltrated the domain completely

Finally, also the average saturation will be used, which follows directly from its definition of being the volume of the fluid phase divided by the volume of the pore space. Thus,

$$
\left\langle S_{\alpha}\right\rangle=\frac{1}{\int_{V} \mathrm{~d} V} \int_{V} S_{\alpha} \mathrm{d} V \quad \alpha=\mathrm{w}, \mathrm{n}
$$

assuming constant porosity.

\section{Model Description}

\subsection{Pressure Distribution Across the Domain}

We will consider a two-phase flow problem, consisting of static primary drainage in a onedimensional homogeneous vertical domain. As the domain is vertical, gravity forces will influence the pressure and saturation distributions. The domain is initially fully saturated with the water phase. Primary drainage is initiated by forcing the nonwetting phase into the domain from below. For a given overpressure at the bottom boundary, pressure and saturation distributions can be obtained assuming static equilibrium (i.e., no flow of both wetting and nonwetting phases). A schematic representation of pressure distributions, for the case that the nonwetting phase density is higher than the wetting phase density, is shown in Fig. 2.

At static equilibrium, the wetting phase pressure distribution will be hydrostatic. Assuming zero pressure at the top of the domain, the pressure distribution is given by:

$$
P_{\mathrm{w}}(z)=\rho_{\mathrm{w}} g(H-z) \quad 0 \leq z \leq H,
$$

where $g$ is the gravitational constant and $\rho_{\mathrm{w}}$ is the wetting phase density. This expression can be made dimensionless by dividing both sides by $\rho_{\mathrm{w}} g H$ :

$$
P_{\mathrm{w}}^{\prime}\left(z^{\prime}\right)=1-z^{\prime} \quad 0 \leq z^{\prime} \leq 1,
$$


where

$$
P_{\mathrm{w}}^{\prime}=\frac{P_{\mathrm{w}}}{\rho_{\mathrm{w}} g H} \quad \text { and } \quad z^{\prime}=\frac{z}{H} .
$$

The nonwetting phase pressure distribution is more complex. When we increase the bottom boundary pressure in steps, the nonwetting phase will infiltrate further into the domain. For each nonwetting phase bottom boundary overpressure $\Delta P$, a particular static equilibrium pressure and saturation distribution can be derived. A distinct infiltration front is present as long as the nonwetting phase has not reached the top of the sample. Below the infiltration front, the pressure distribution is given by:

$$
P_{\mathrm{n}}(z)=\rho_{\mathrm{w}} g H+P_{\mathrm{d}}+\Delta P-\rho_{\mathrm{n}} g z \quad 0 \leq z \leq z_{f},
$$

where $P_{\mathrm{d}}$ is the entry pressure of the porous medium, $\rho_{\mathrm{n}}$ is the nonwetting phase density, and $z_{f}$ is the height of the infiltration front. The height of the infiltration front $z_{f}$ is given by:

$$
z_{f}=\frac{\Delta P}{\left(\rho_{\mathrm{n}}-\rho_{\mathrm{w}}\right) g} .
$$

Making Eq. 3.4 dimensionless, we obtain:

$$
P_{\mathrm{n}}^{\prime}\left(z^{\prime}\right)=1+P_{\mathrm{d}}^{\prime}+\Delta P^{\prime}-\rho^{\prime} z^{\prime} \quad 0 \leq z^{\prime} \leq z_{f}^{\prime}
$$

where

$$
P_{\mathrm{n}}^{\prime}=\frac{P_{\mathrm{n}}}{\rho_{\mathrm{w}} g H} \quad P_{\mathrm{d}}^{\prime}=\frac{P_{\mathrm{d}}}{\rho_{\mathrm{w}} g H} \quad \Delta P^{\prime}=\frac{\Delta P}{\rho_{\mathrm{w}} g H} \quad \rho^{\prime}=\frac{\rho_{\mathrm{n}}}{\rho_{\mathrm{w}}} \quad z_{f}^{\prime}=\frac{z_{f}}{H}=\frac{\Delta P^{\prime}}{\rho^{\prime}-1} .
$$

In the remainder of this article, the prime sign will be omitted, but it must be understood that all variables are dimensionless unless stated otherwise.

Above the infiltration front, in the case of primary drainage, the nonwetting phase is not present. However, the simple average pressure averages over the whole domain and, therefore, a pressure distribution needs to be assumed. Here, we assume that when a phase is not present, its pressure still is defined based on the Brooks-Corey relationship. According to the Brooks-Corey relationship, at the wetting saturation of unity, we may assume that the difference between the nonwetting and wetting phase pressures is equal to the local entry pressure. This is a common assumption in most numerical models (e.g., STOMP, White and Oostrom 1997; MUFTE-UG, Helmig 1997). Thus, we assume the dimensionless pressure distribution above the infiltration front is given by:

$$
P_{\mathrm{n}}(z)=1-z+P_{\mathrm{d}} \quad z_{f}<z \leq 1 .
$$

The saturation distribution can be derived from the nonwetting and wetting phase pressure distribution through the Brooks-Corey relationship:

$$
\begin{array}{ll}
S_{\mathrm{W}}(z)=\left(1-S_{\mathrm{wr}}-S_{\mathrm{nr}}\right)\left(\frac{P_{\mathrm{n}}(z)-P_{\mathrm{w}}(z)}{P_{\mathrm{d}}}\right)^{-\lambda}+S_{\mathrm{wr}} & 0 \leq z \leq z_{f} \\
S_{\mathrm{W}}(z)=1 & z_{f} \leq z \leq 1,
\end{array}
$$

where $\lambda$ is the pore-size distribution coefficient, $S_{\mathrm{wr}}$ is the residual wetting phase saturation, and $S_{\mathrm{nr}}$ is the residual nonwetting phase saturation. Table 1 contains the values for the different parameters used in this article.

From the static equilibrium pressure and saturation distributions, average values can be determined using the averaging operators introduced in Sect. 2. This can be done for a range 
Table 1 Parameters used in this article

Brooks-Corey parameters

Dimensionless entry pressure $\left(P_{\mathrm{d}}\right)$

Pore-size distribution index $(\lambda)$

6.11

Residual wetting phase saturation $\left(S_{\mathrm{wr}}\right)$

0.10

Residual nonwetting phase saturation $\left(S_{\mathrm{nr}}\right)$ primary drainage

Residual nonwetting phase saturation $\left(S_{\mathrm{nr}}\right)$ main drainage

0

Phase densities

Density ratio $\rho$

of values of $\Delta P$. Note that for the one-dimensional dimensionless domain, integration is not performed over volume $V$, but from zero to one.

\section{Results and Discussion}

\subsection{The Correct Average Pressure}

Before comparing the different averaging operators, we need to determine what the correct average pressure actually should be. This can be done by considering the phase potentials:

$$
\begin{aligned}
& \Phi_{\mathrm{n}}(z)=P_{\mathrm{n}}+\rho z \\
& \Phi_{\mathrm{w}}(z)=P_{\mathrm{w}}+z .
\end{aligned}
$$

As static equilibrium is assumed, the phase potentials must be constant throughout the domain, and equal to their boundary values. These constant potentials at $z=0$ are found to be equal to:

$$
\begin{aligned}
& \Phi_{\mathrm{n}}=1+P_{\mathrm{d}}+\Delta P \\
& \Phi_{\mathrm{w}}=1 .
\end{aligned}
$$

The average of a constant local potential should simply be equal to the local potential:

$$
\begin{aligned}
& \left\langle\Phi_{\mathrm{n}}\right\rangle=\left\langle P_{\mathrm{n}}\right\rangle+\rho\langle z\rangle=\Phi_{\mathrm{n}} \\
& \left\langle\Phi_{\mathrm{w}}\right\rangle=\left\langle P_{\mathrm{w}}\right\rangle+\langle z\rangle=\Phi_{\mathrm{w}} .
\end{aligned}
$$

In our dimensionless domain, $\langle z\rangle$ is equal to 1/2. Based on Eqs. 4.2 and 4.3, the correct average pressure thus is given by:

$$
\begin{aligned}
& \left\langle P_{\mathrm{n}}\right\rangle=\left\langle\Phi_{\mathrm{n}}\right\rangle-\rho\langle z\rangle=1+P_{\mathrm{d}}+\Delta P-\frac{1}{2} \rho \\
& \left\langle P_{\mathrm{w}}\right\rangle=\left\langle\Phi_{\mathrm{w}}\right\rangle-\langle z\rangle=\frac{1}{2} .
\end{aligned}
$$

In the remainder of this article, these average pressures are referred to as the potential-based average pressures. They represent the correct average pressures because:

(1) they preserve the local constant phase potentials at the upscaled level, and

(2) they represent the pressure at the centroid of the domain, $\langle z\rangle$. 


\subsection{Average Pressure During Primary Drainage}

In this section, the averaging operators introduced in Sect. 2 are compared to each other and to the potential-based average pressure for primary drainage. We consider the simple case, where throughout the primary drainage process, the local nonwetting phase pressure is larger than the local wetting phase pressure when extrapolated in a linear fashion above the infiltration front. The resulting pressure distribution for one particular situation in the primary drainage process $(\Delta P=0.25)$ and the corresponding average pressures are plotted in Fig. 3.

In this particular case, the nonwetting phase front has reached approximately 0.4 of the total domain height. As can be seen, the wetting phase averages have identical values, because the wetting phase is present throughout the domain. An exception to this is the wetting intrinsic phase-volume average pressure, which underestimates the average pressure. This is because the local wetting phase pressures are weighted with the wetting phase saturation, which is lower at lower elevations where pressure is high. As a result, the lower pressures higher in the domain are assigned more weight. For a similar reason, the nonwetting intrinsic phasevolume average pressure overestimates the average nonwetting phase pressure. As can be seen from Fig. $3 \mathrm{~b}$ as well, the centroid of the wetting phase, to which the wetting intrinsic phase-volume average pressure is assigned, lies above the centroid of the domain. Similarly, the centroid of the nonwetting phase lies far below the centroid of the domain. All averaging operators, except the centroid-corrected phase-average pressure, give a different nonwetting phase-average pressure than the potential-based average pressure.

Figure 3 only shows the difference between the average pressures for one particular average saturation (i.e., one moment in the drainage process). In order to see how the average pressures evolve through drainage steps, the average pressures are plotted as a function of average saturation as shown in Fig. 4.

As can be seen, the differences in average pressure for the different averaging operators become smaller as the drainage process progresses. The largest differences between averaging operators are found for the nonwetting phase, as it is initially not present everywhere in the domain. For the wetting phase, the average pressures are all equal to each other and constant throughout the drainage process, except for the intrinsic phase-volume average pressure.

Figure 5 shows the centroids of the phases and the centroid of the domain as a function of average water saturation. Clearly, the centroid of the nonwetting phase moves up as the nonwetting phase front invades the domain. The centroid of the nonwetting phase does not coincide with the centroid of the domain even when the nonwetting phase reaches the outflow of the domain (this occurs at $\left\langle S_{\mathrm{w}}\right\rangle=0.48$ ), because the saturation continues to be nonlinear. Only once the nonwetting phase saturation becomes uniform, the phase centroid reaches the middle of the domain. The centroid of the wetting phase coincides initially with the centroid of the domain, as the domain is completely filled with water. However, during the drainage process, the wetting phase centroid moves up because the nonwetting phase replaces the wetting phase from below. At higher elevations, the water saturation is higher, and as a result, the wetting phase centroid is situated above the middle of the domain. The maximum height of the wetting phase centroid corresponds to the nonwetting phase reaching the top of the domain. After that the wetting phase distribution becomes more and more uniform and the wetting phase centroid approaches the centroid of the domain.

Now, we return to the two requirements for a correct pressure (at static conditions) described in Sect. 4.1. The average potentials using the various averaging operators can be calculated according to:

$$
\left\langle\Phi_{\mathrm{n}}\right\rangle^{a}=\left\langle P_{\mathrm{n}}\right\rangle^{a}+\rho\left\langle z_{\mathrm{n}}\right\rangle^{a},
$$



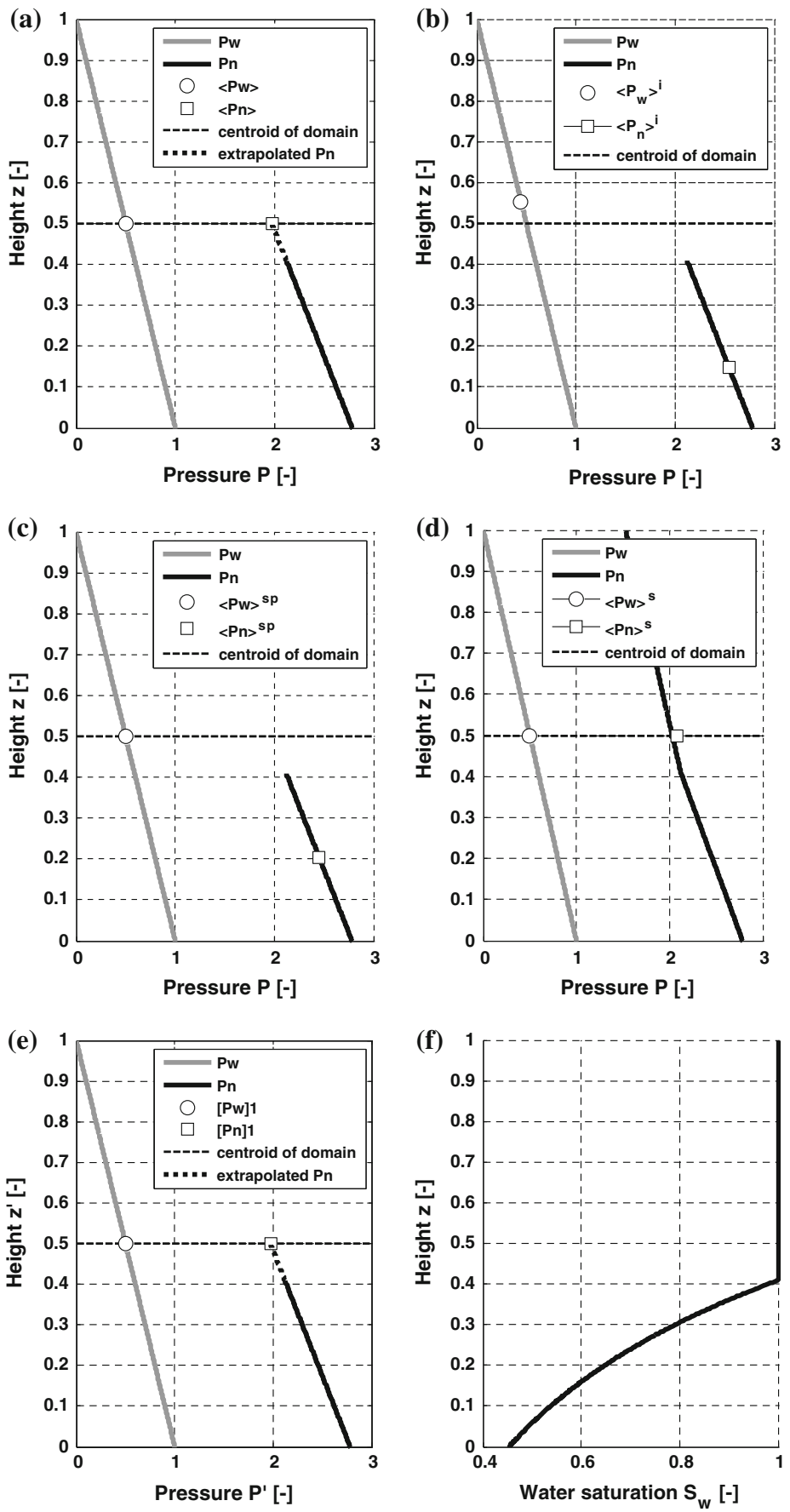

Fig. 3 Pressure and saturation distributions used to obtain average pressures for $\Delta P=0.25$ and $\rho=1.6$. a Potential-based average pressure; $\mathbf{b}$ intrinsic phase-volume average pressure; $\mathbf{c}$ simple phase-average pressure; $\mathbf{d}$ simple average pressure; e centroid-corrected phase-average pressure; $\mathbf{f}$ saturation distribution 


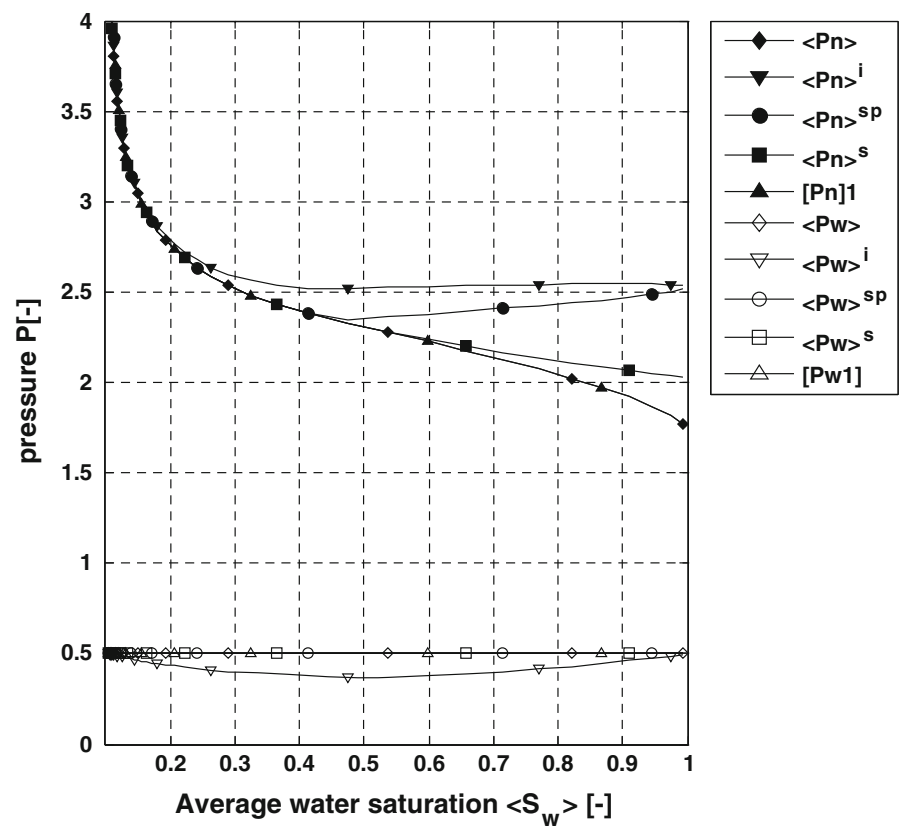

Fig. 4 Average pressures as a function of average saturation (a measure for the progress of the drainage process) for primary drainage

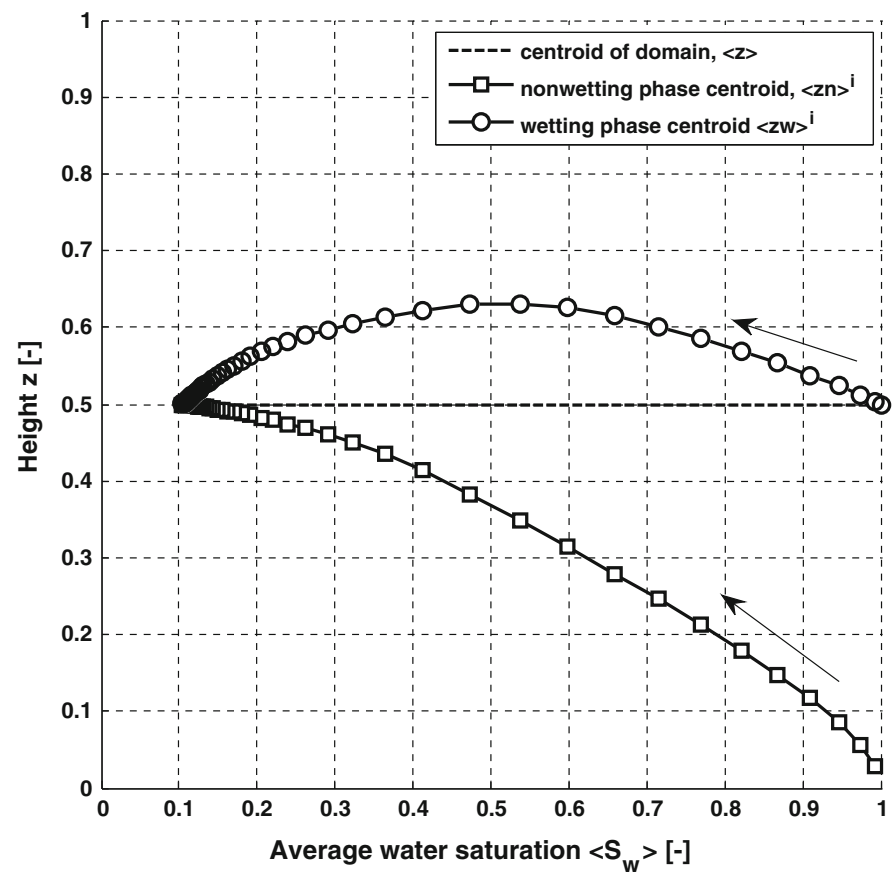

Fig. 5 Centroid of the domain and centroids of the phases as a function of average water saturation $\left\langle S_{\mathrm{W}}\right\rangle$ during primary drainage 
where the superscript $a$ refers to one of the averaging operators. The average coordinate $\langle z\rangle^{a}$ can be calculated in the same manner as the average pressure, for instance, the intrinsic phase-volume average coordinate is defined as:

$$
\left\langle z_{\alpha}\right\rangle^{i}=\frac{1}{\int_{V} S_{\alpha} \mathrm{d} V} \int_{V} S_{\alpha} z \mathrm{~d} V \quad \alpha=\mathrm{w}, \mathrm{n} .
$$

Note that $\left\langle z_{\alpha}\right\rangle^{i}$ can have different values for the wetting and the nonwetting phase. Thus, a correct average operator is the one which, after calculating $\left\langle P_{\mathrm{n}}\right\rangle^{a}$ and $\left\langle z_{\mathrm{n}}\right\rangle^{a}$ and substituting these into Eq. 4.5, gives an average potential that is equal to the local constant potential. In addition, $\left\langle z_{\mathrm{n}}\right\rangle^{a}$ should be equal to the centroid of the domain, which in this case is 0.5 . The potential-based average operator fulfills these requirements, as does the centroid-corrected phase-volume average operator. The other averaging operators, however, do not meet both requirements. The intrinsic phase-volume average operator does give an average potential that is equal to the local constant potential, but the average coordinate is not at the middle of the domain. The same holds for the simple phase-average operator. The simple average pressure, although returning the pressure at the middle of the domain, does not give the correct average potential.

The intrinsic phase-volume average and simple phase average can be corrected such that the correct average pressure is obtained. Consider that for any averaging operator, preserving the local constant potential, the following holds:

$$
\left\langle\Phi_{\alpha}\right\rangle^{a}=\left\langle P_{\alpha}\right\rangle^{a}+\rho\left\langle z_{\alpha}\right\rangle^{a} .
$$

Moreover, all the average potentials are equal to the constant local potential and therefore to each other:

$$
\left\langle\Phi_{\alpha}\right\rangle^{i}=\left\langle\Phi_{\mathrm{a}}\right\rangle^{s p}=\left\langle\Phi_{\mathrm{a}}\right\rangle=\Phi_{\alpha} .
$$

Using these relations, it is possible to rewrite the average pressures into each other. For the intrinsic phase-volume average pressure, we obtain:

$$
\left\langle P_{\alpha}\right\rangle=\left\langle P_{\alpha}\right\rangle^{i}+\rho\left(\left\langle z_{\alpha}\right\rangle^{i}-\langle z\rangle\right) .
$$

As this equation shows, the correct pressure is obtained by correcting the intrinsic phasevolume average pressure for the difference between the centroid of the average coordinate $\left\langle z_{\alpha}\right\rangle^{i}$ and the averaging domain $\langle z\rangle$. Actually, this is also exactly what the centroid-corrected phase average does. Equation 4.9 is a special case of Eq. 2.5, where:

$$
\frac{\frac{\partial}{\partial z}\left\langle P_{\alpha}\right\rangle^{i}}{\frac{\partial}{\partial z}\left\langle z_{\alpha}\right\rangle^{i}}=-\rho .
$$

A similar expression can be obtained for the simple phase-average pressure. In fact, the correct pressure can be obtained from any averaging operator preserving the local constant potential using expressions similar to Eq. 4.9. The simple average cannot be corrected this way, because it does not preserve the local constant potential and therefore requirement (4.8) cannot be satisfied.

The relation between the intrinsic phase-volume average pressure and the potential-based average pressure pressure given in Eq. 4.9 is only valid for equilibrium conditions. However, it is important to note that the definition given by Nordbotten et al. (2008), which was called centroid-corrected average pressure in this article, holds for nonequilibrium too. Therefore, it is the preferred averaging operator to use. 

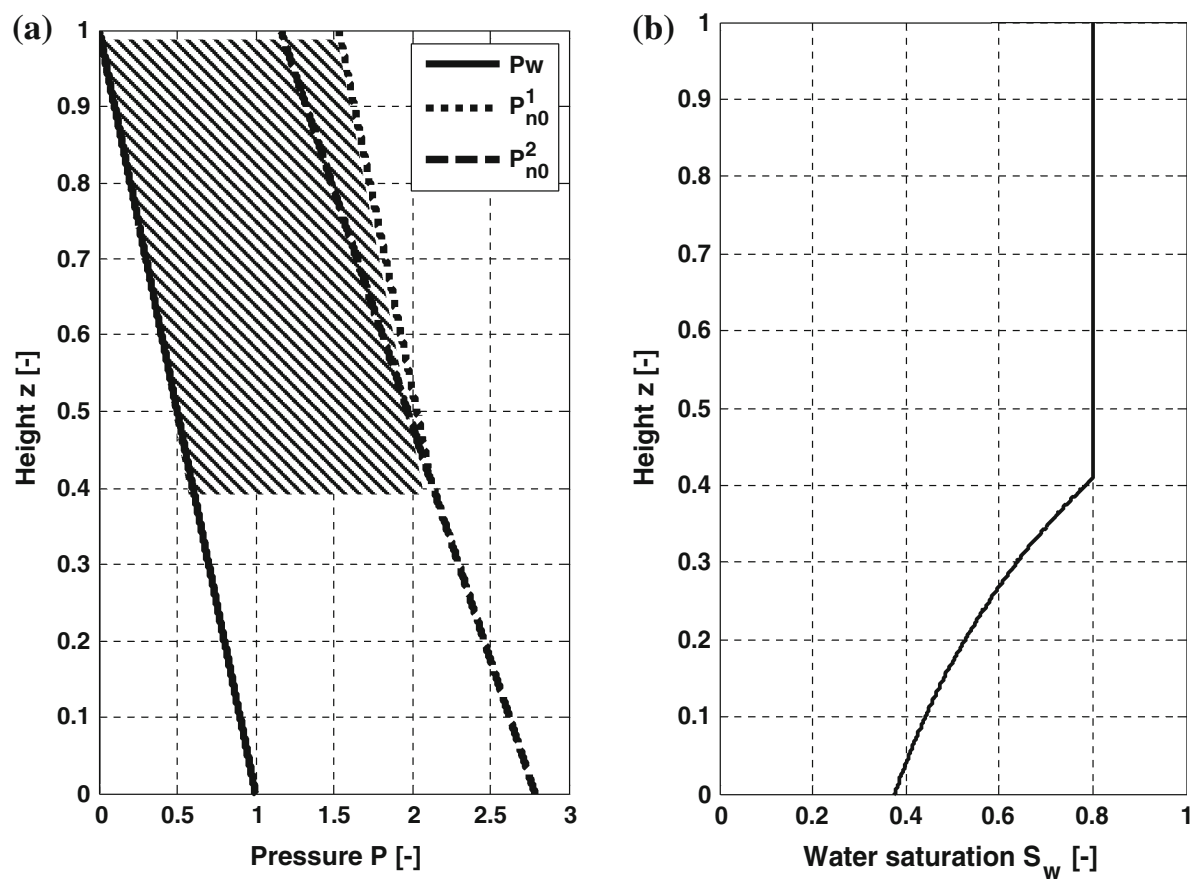

Fig. 6 a Choice of pressure distribution above infiltration front. The shaded area corresponds to $P_{\mathrm{n} 0}^{1}$, which can be anything between $P_{\mathrm{W}}$ and $P_{\mathrm{W}}+P_{\mathrm{d}}$. However, here, we assume $P_{\mathrm{n} 0}^{1}=P_{\mathrm{W}}+P_{\mathrm{d}}$ above the infiltration front. b Corresponding saturation distribution

\subsection{Average Pressure During Main Drainage}

During primary drainage, the nonwetting phase initially is not present everywhere. As a result, the centroids of the phases are different from each other and from the centroid of the domain. This difference is largest in the primary drainage process. Considering a main drainage process, on the other hand, where the nonwetting phase is already present throughout the domain (though at residual saturation), the differences between the centroids of the phases and the centroid of the domain are expected to be less.

The pressure distributions in this case are equal to the pressure distributions introduced in Eq. 3.2 and 3.6 for primary drainage. However, now above the infiltration front, the nonwetting phase is already present at residual saturation. Therefore, the choice of the nonwetting phase pressure distribution above the front is important for all averaging operators.

We have two choices for the nonwetting phase pressure distribution above the infiltration front during main drainage. The first is to assume that the nonwetting phase above the infiltration front is residual, but continuous. In this case, the pressure distribution can be linearly extrapolated from the pressure below the front (see Fig. 6, $P_{\mathrm{n} 0}^{2}$ ). Alternatively, if we assume that the nonwetting phase is discontinuous, we may choose anything for the nonwetting phase pressure in between $P_{\mathrm{w}}$ and $P_{\mathrm{w}}+P_{\mathrm{d}}$. In this study, we assume the nonwetting phase pressure above the front is equal to $P_{\mathrm{w}}+P_{\mathrm{d}}$ (see Fig. 6, $P_{\mathrm{n} 0}^{1}$ ). We consider both choices for the calculation of average pressures.

Figure 7 shows the obtained average pressures as a function of average saturation, assuming the nonwetting phase pressure distribution $P_{\mathrm{n} 0}^{1}$. The difference between the various 


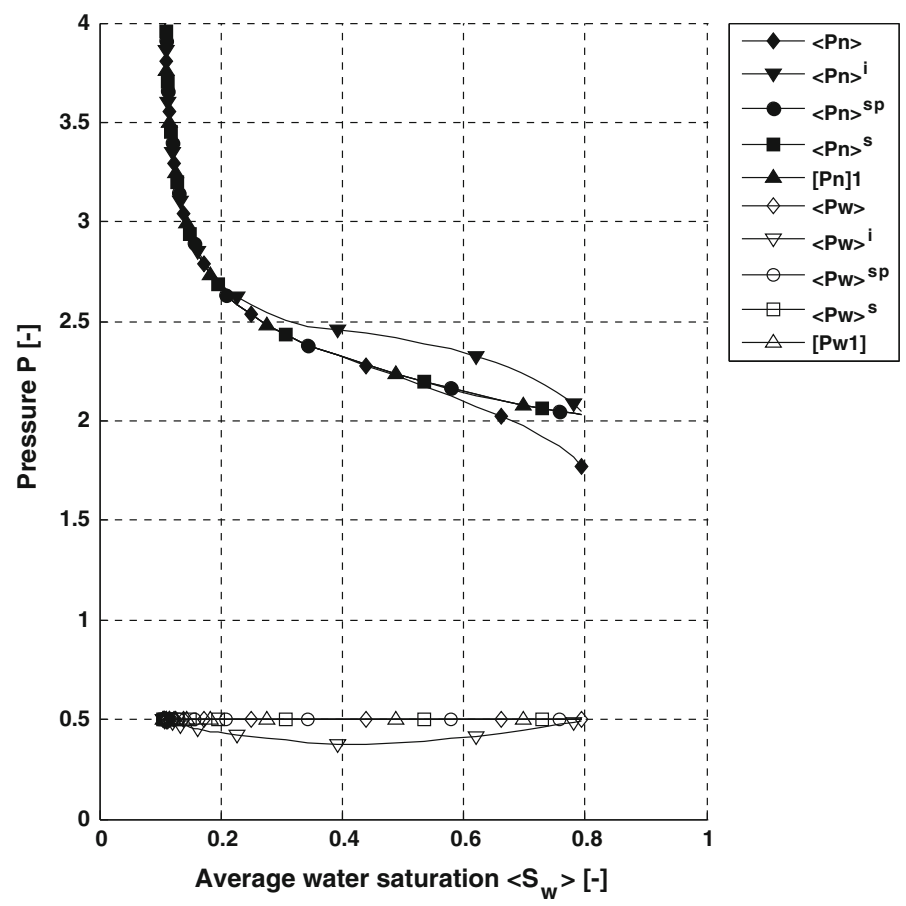

Fig. 7 Average pressures as a function of average, for main drainage, assuming $P_{\mathrm{n} 0}^{1}\left(P_{\mathrm{n}}=P_{\mathrm{W}}+P_{\mathrm{d}}\right.$ above $z_{f}$ )

average pressures is now significantly less than that it was for primary drainage (compare with Fig.4). In fact, the curves for the nonwetting simple phase-average pressure and the nonwetting centroid-corrected average pressure now coincide with the nonwetting simple average pressure curve. This is, of course, because now the same pressure distribution and the same averaging domain are used in calculating the average pressures. The intrinsic phasevolume average pressure is still different from the other averages because it weighs pressure with saturation. Note that when pressure distribution $P_{\mathrm{n} 0}^{1}$ is used, the local potential is not constant anymore, because the pressure distribution has become nonlinear (there is a kink at the infiltration front). This is further discussed in the next section. For the wetting phaseaverage pressure, nothing has changed because the pressure distribution stays the same (i.e., hydrostatic) for both primary and main drainage.

Next consider $P_{\mathrm{n} 0}^{2}$, the second choice for the nonwetting phase pressure distribution suggested in Fig. 6. The resulting average pressures are plotted as a function of average saturation in Fig. 8.

Again, the wetting phase pressures are similar to the primary drainage case. However, now the curves for nonwetting centroid-corrected average pressure and the potential-based average pressure coincide, as it was the case for primary drainage. This is because the assumed nonwetting phase pressure distribution for main drainage is equal to the nonwetting phase pressure when it is extrapolated to the centroid of the domain in the case of primary drainage.

Moreover, both curves coincide with curves for the simple phase average and simple average pressures.

The differences between the centroids of the phases and the middle of the domain are also smaller in the case of main drainage, as shown in Fig. 9 (compare to Fig. 5). 


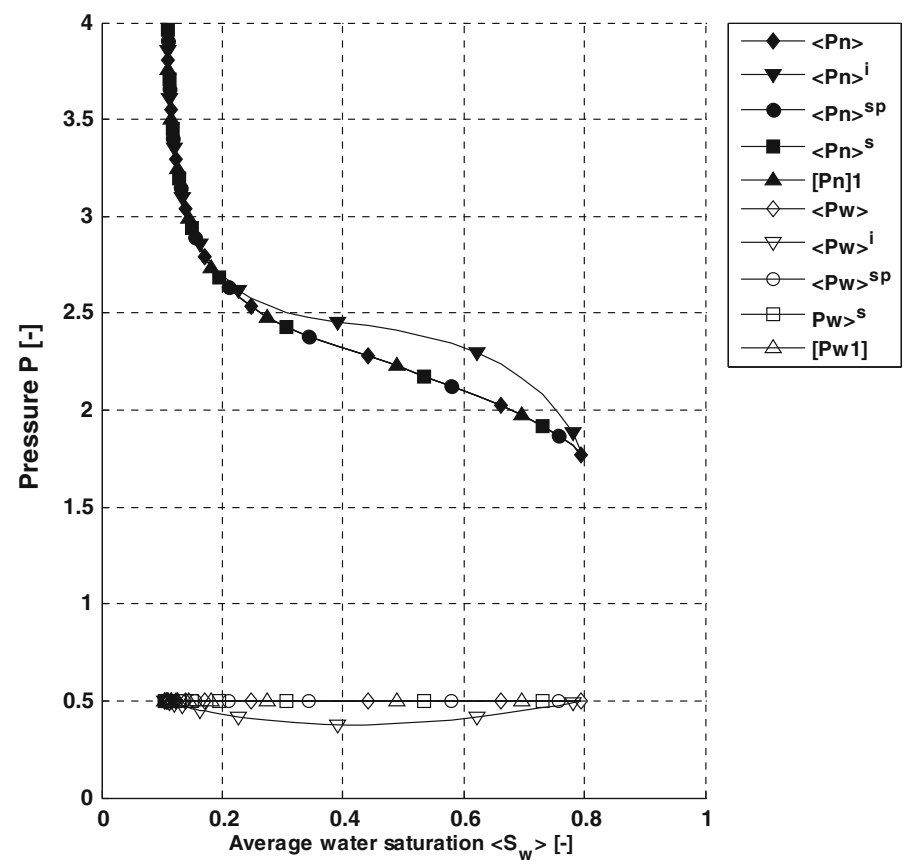

Fig. 8 Average pressures as a function of average saturation, for main drainage, assuming $P_{n 0}^{2}$

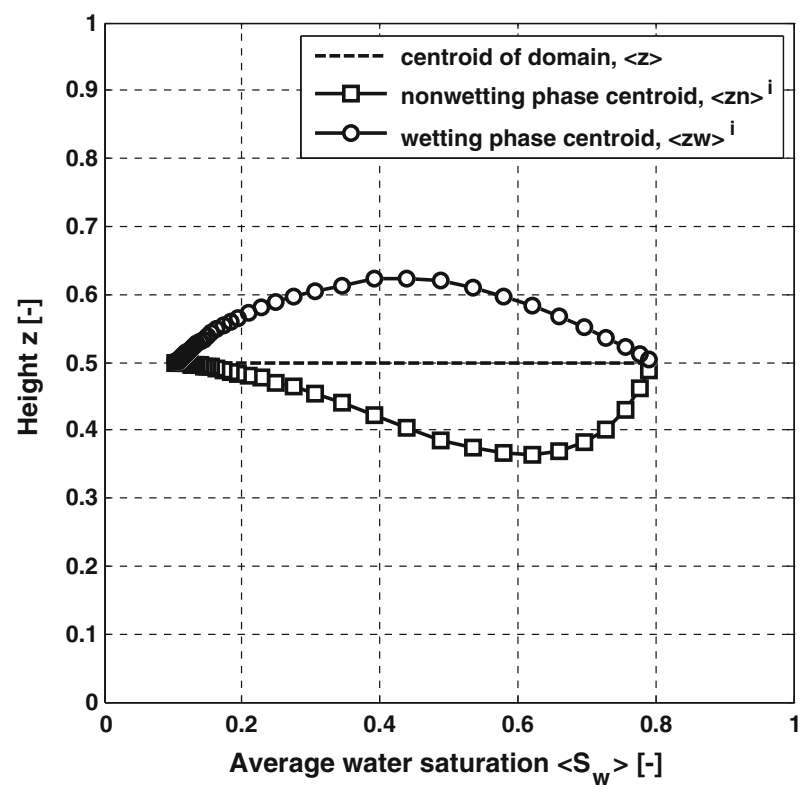

Fig. 9 Centroid of the domain and centroids of the phases as a function of average water saturation $\left\langle S_{\mathrm{W}}\right\rangle$ during main drainage 


\subsection{Consequences of the Choice of $\mathrm{P}_{n 0}$ Above the Infiltration Front for Main Drainage}

In the previous section, we assumed two different nonwetting phase pressure distributions above the infiltration front, $P_{\mathrm{n} 0}^{1}$ and $P_{\mathrm{n} 0}^{2}$. They gave different results for several averaging operators. However, what is the consequence of using either of these distributions?

First, consider the pressure distribution $P_{n 0}^{2}$, where the nonwetting phase pressure is extrapolated in a linear fashion from the nonwetting phase distribution below the front (see Fig. 6). This assumption for the pressure distribution above the front conserves the local constant potential at the average scale, because the pressure distribution is linear throughout. At the same time, this assumption can cause the nonwetting phase pressure (both local and average) to become smaller than the wetting phase pressure, which results in a negative capillary pressure. In the examples discussed above, this did not happen because for this combination of domain length and boundary pressures, the (extrapolated) nonwetting phase pressure was larger than the wetting phase pressure at all times.

However, there are situations where the centroid-corrected capillary pressure (and the potential-based average pressure as well) will become negative. This occurs, for example, when the domain is very long, the entry pressure is small, or the density ratio $\rho_{\mathrm{n}} / \rho_{\mathrm{w}}$ is large. In fact, this may occur (at least at some stage during the drainage process) when:

$$
\Delta P+P_{\mathrm{d}}+\frac{1}{2}(1-\rho)<0 .
$$

Note that the domain height is implicitly included in these dimensionless variables.

Whenever inequality (4.11) is met, the nonwetting centroid-corrected phase average- and potential-based phase-average pressure will be smaller than the wetting phase-average pressure:

$$
\left\langle P_{\mathrm{c}}\right\rangle=\left\langle P_{\mathrm{n}}\right\rangle-\left\langle P_{\mathrm{w}}\right\rangle<0 .
$$

This occurs for small values of $\Delta P$ (how small depends on the values of $P_{\mathrm{d}}$ and $\rho$ ). At some point in the drainage process, $\Delta P$ becomes large enough to make the left-hand side of Eq. 4.12 positive. Thus, the average capillary pressure would be negative early in the drainage process. This is illustrated in Fig. 10, for a situation where the dimensionless entry pressure $P_{\mathrm{d}}$ was reduced from 1.53 (used in the examples up till now) to 0.06 . Reducing this parameter either means that the entry pressure is decreased, or the domain height is increased. By increasing the density ratio $\rho_{\mathrm{n}} / \rho_{\mathrm{W}}$, similar results are obtained.

It is evident that for $P_{\mathrm{d}}=0.06$, the average capillary pressures are all negative for large values of $\left\langle S_{\mathrm{w}}\right\rangle$. Once the infiltration front has reached the centroid of the domain (at approximately $\left.\left\langle S_{\mathrm{w}}\right\rangle=0.57\right)$, the potential-based and centroid-corrected average capillary pressure become positive again.

Although using pressure distribution $P_{\mathrm{n} 0}^{2}$ above the infiltration front does conserve local constant potential, the occurrence of negative capillary pressures when residual nonwetting phase is present is not physically correct.

Next, consider the pressure distribution $P_{\mathrm{n} 0}^{1}$. In this case, the nonwetting phase pressure remains larger than the wetting phase pressure throughout the domain and at all stages. As a result, capillary pressure would also remain positive. However, now the local nonwetting phase potential is no longer constant, because the nonwetting phase pressure distribution is nonlinear (as long as the front has not reached the upper boundary). The advantage of using $P_{\mathrm{n} 0}^{1}$ is that it gives a physically acceptable nonwetting phase pressure distribution when that phase is present at residual saturation. A disadvantage of this assumption arises when two different (averaging) domains on top of each other are considered (similar to two adjacent 

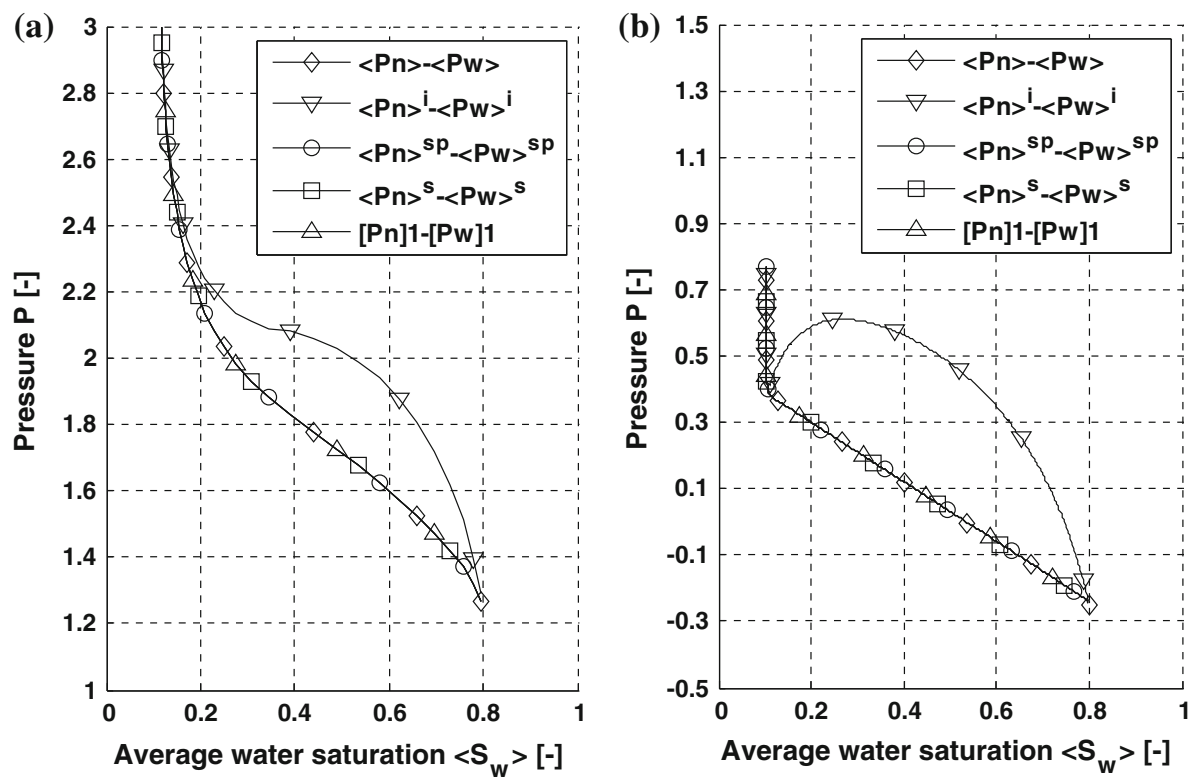

Fig. 10 a $\left\langle P_{\mathrm{n}}\right\rangle-\left\langle P_{\mathrm{W}}\right\rangle$ versus $\left\langle S_{\mathrm{W}}\right\rangle$ for the main drainage process, using nonwetting phase pressure distribution $P_{\mathrm{n} 0}^{2}$, and a dimensionless entry pressure of 1.53 ; $\mathbf{b}$ the same, but with a dimensionless entry pressure of 0.06

(a)

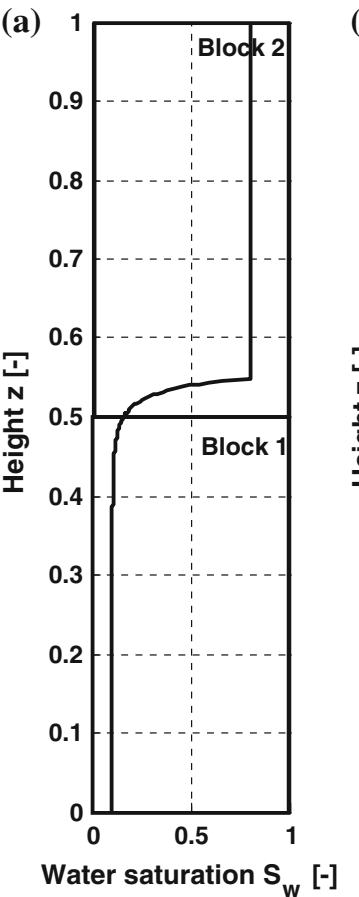

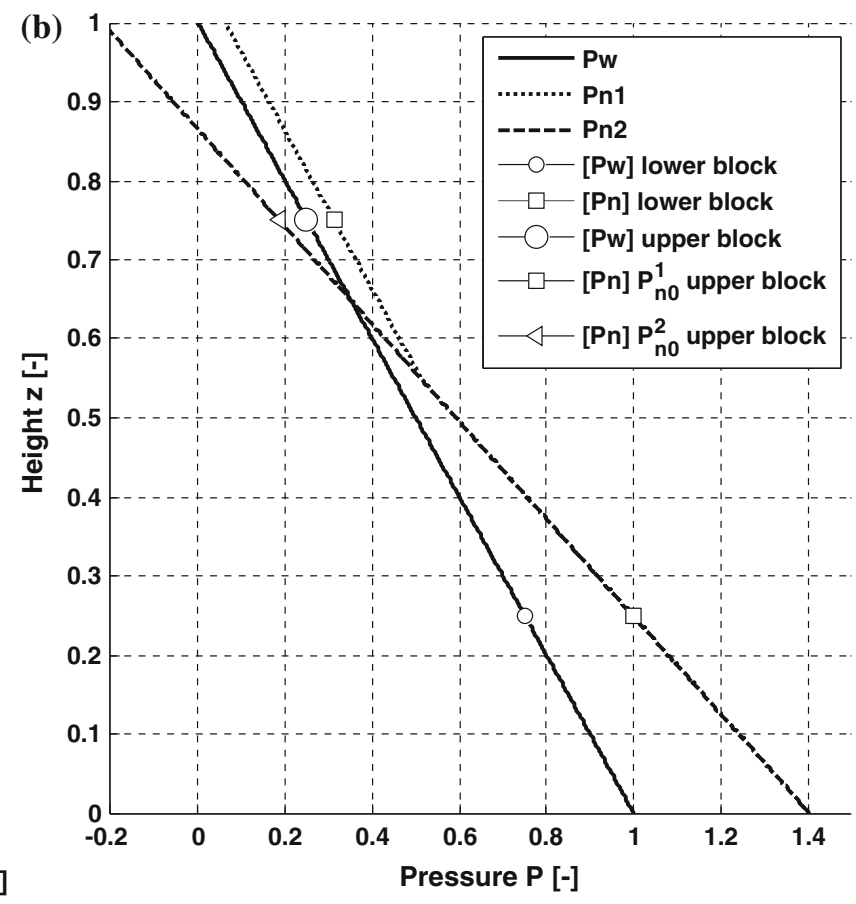

Fig. 11 a Saturation distribution for two identical domains placed on top of each other. b Corresponding pressure distribution and average pressures 
Table 2 Average values for several variables in the two grid blocks shown in Fig. 11

\begin{tabular}{lllll}
\hline Average values & \multicolumn{2}{l}{ Lower domain } & \multicolumn{2}{l}{ Upper domain } \\
\hline$\left\langle\Phi_{\mathrm{n}}\right\rangle$ & 1.40 & & 1.53 & \\
$\left\langle\Phi_{\mathrm{w}}\right\rangle$ & 1 & & 1 & \\
$\left\langle S_{\mathrm{W}}\right\rangle$ & 0.11 & & 0.75 & \\
$\left\langle S_{\mathrm{n}}\right\rangle$ & 0.89 & & 0.25 & \\
Pressure distribution & $P_{\mathrm{n} 0}^{1}$ & $P_{\mathrm{n} 0}^{2}$ & $P_{\mathrm{n} 0}^{1}$ & $P_{\mathrm{n} 0}^{2}$ \\
{$\left[P_{\mathrm{n}}\right]-\left[P_{\mathrm{W}}\right]$} & 0.25 & 0.25 & 0.06 & -0.06 \\
\hline
\end{tabular}

grid blocks in a numerical simulator). Figure 11 illustrates such a situation, where, in a main drainage situation, the infiltration front is beyond the lower domain, but still somewhere in the upper domain. Thus, the upper part of the upper block is still at residual nonwetting phase saturation. Table 2 gives the averages calculated for the particular situation of Fig. 11. When the average potential for each block is calculated, we find:

$$
\left\langle\Phi_{\mathrm{n}}\right\rangle^{\text {upper }}>\left\langle\Phi_{\mathrm{n}}\right\rangle^{\text {lower }} .
$$

This means that there is a difference in the potential between the two domains under static conditions, which is a nonphysical result.

Concluding this section, for main drainage, we have two choices for the nonwetting phase pressure distribution above the infiltration front. Assuming the pressure distribution is a linear extrapolation of the nonwetting phase pressure below the front, the constant-potential criterion is satisfied, but under certain conditions (small entry pressure, large domain length, and/or large density ratio), the (extrapolated) local and average capillary pressures can become negative. When it is assumed that the nonwetting phase pressure distribution above the front is equal to $P_{\mathrm{w}}+P_{\mathrm{d}}$, we obtain physically acceptable local and average capillary pressures, but then the constant-potential criterion is violated.

\subsection{When is the Use of a Correct Averaging Operator Important?}

One could ask the question when the difference between various averaging operators is significant? For instance, when the averaging domain is sufficiently small, a front will only be present in the very early stages of the drainage process, and saturation will be more or less constant throughout the domain. In such a case, all averaging operators will give the same average pressure within a certain range of error. Also, as shown here, when both phases are initially present (as in main drainage or main imbibition situations), differences among various averaging operators are small. Now, even for the case of primary drainage, when the difference between the centroids of the phase and the centroid of the averaging domain becomes sufficiently small, the averaging operators all give the same result. Thus, we need to develop a criterion in this regard. One possible criterion is the dimensionless number $N_{\mathrm{d}}$ :

$$
N_{\mathrm{d}}=\rho\left(\left\langle z_{\mathrm{n}}\right\rangle^{a}-\langle z\rangle\right)+\left(\left\langle z_{\mathrm{w}}\right\rangle^{a}-\langle z\rangle\right) .
$$

When this number is sufficiently small, effects due to choice of the averaging operator may be neglected. Alternatively, another dimensionless number can be introduced:

$$
N_{\mathrm{g}}=\frac{(1-\rho) \hat{\mathbf{e}}_{f} \cdot \hat{\mathbf{e}}_{\mathrm{g}}}{P_{\mathrm{d}}}
$$




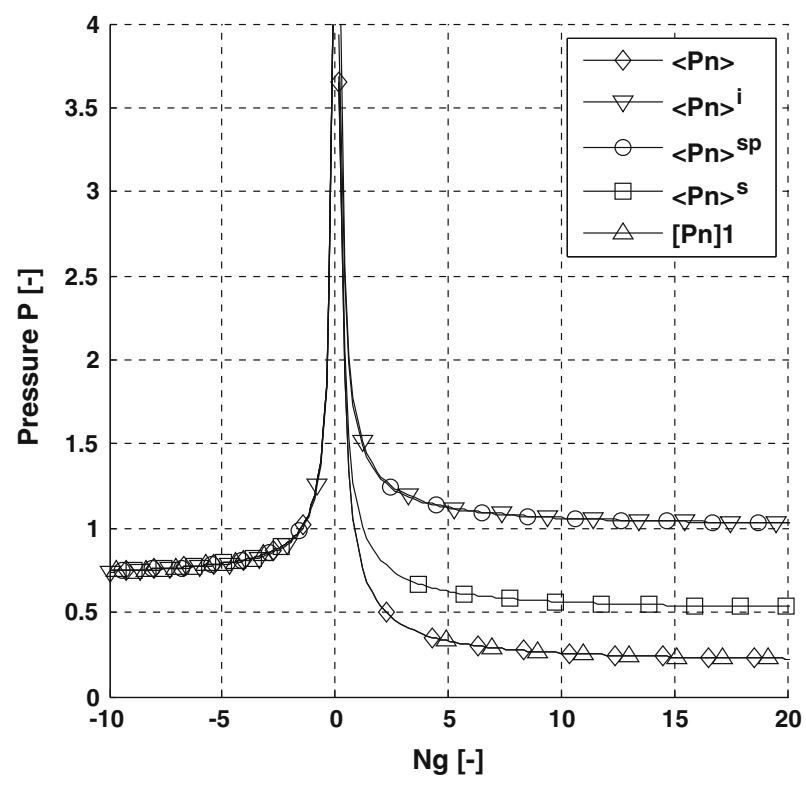

Fig. 12 Values for the different nonwetting phase-average pressures as a function of $N_{\mathrm{g}}$

where $\hat{\mathbf{e}}_{f}$ is the unit vector in the direction of flow relative to the gravitational constant, and $\hat{\mathbf{e}}_{z}$ is the gravitational unit vector. When $N_{\mathrm{g}}$ approaches zero or becomes negative, the difference between various averaging operators can be neglected, because front formation will not readily occur. However, when it becomes much larger than zero, care should be taken to use the correct averaging operator. Figure 12 shows the values of different average pressures as a function of $N_{\mathrm{g}}$. Clearly, differences are largest for the nonwetting phase pressure when $N_{\mathrm{g}}>0$.

Finally, note that the case discussed in this article is a specific case where a dense nonwetting phase fluid replaces a lighter wetting phase fluid from below. In this case, a front will exist, and $N_{\mathrm{g}}$ will be positive. The equations (such as Eq. 4.11) introduced in this article only apply to this situation. However, a similar situation arises when a nonwetting phase fluid, which is lighter than the wetting phase fluid (i.e., $\rho<1$ ), is forced to replace the wetting phase fluid from above. In that case, there will be a front as well and $N_{\mathrm{g}}$ will be positive. Alternatively, consider the case where a lighter nonwetting phase fluid is forced into the domain from below, or a denser nonwetting phase fluid is forced into the domain from above. In these cases, there will be no clear infiltration front, and $N_{\mathrm{g}}$ is negative, resulting in negligible differences between the average pressures.

\section{Summary and Conclusions}

In this article, several averaging operators are introduced and used to obtain average or upscaled fluid pressures. The most commonly used averaging operator is the intrinsic phasevolume average, which weighs point pressure values with point saturation values. An alternative is the simple phase average, which does not weigh pressure with saturation, but applies an indicator function that ensures that averaging is performed only over the regions where 
the phase under consideration is actually present. Yet another alternative is the simple average, which assumes that the pressure of phase $\alpha$ is defined everywhere in the domain of interest (even where it does not exist) and performs the averaging over the whole domain. The centroid-corrected phase-average pressure, or in short the centroid-corrected pressure was introduced by Nordbotten et al. (2008) as a part of a family of macroscale pressures. This averaging operator corrects the intrinsic phase-volume average pressure for the distance between the centroid of the averaging volume and the phase.

We have studied the differences among these averaging operators by applying them to static equilibrium situations in a vertical homogeneous domain. We consider both primary and main drainage cases, where the nonwetting phase was injected through the bottom boundary. At static equilibrium, pressure and saturation distributions were derived for a given nonwetting phase bottom boundary overpressure $\Delta P$. These distributions were used in the calculation of average pressure and saturation. An important feature of static equilibrium is that the total potential (i.e., sum of pressure and gravity potentials) is constant for each phase. Therefore, its average will be equal to the same constant. Thus, we imposed the criterion that the average phase pressure (which is assigned to the centroid of the averaging volume), plus the gravity potential at the domain centroid must be equal to the constant phase potential for any given conditions.

We have found that the intrinsic phase-volume average pressure, which is commonly employed in averaging studies, results in a gradient in the total phase potential, i.e., the above criterion is violated. In fact, only the centroid-corrected operator satisfies this criterion. However, at high saturations, use of the centroid-corrected average can give rise to negative values of the difference between the average nonwetting and wetting phase pressures.

For main drainage, differences among various averaging operators are significantly less because both phases are present initially, such that the difference between the centroids of phases and the middle of the domain are relatively small. As the nonwetting phase above the infiltration front is present at residual saturation, it is important to make a choice for the nonwetting phase pressure distribution above the front. We may assume that the nonwetting phase pressure above the front either can be extrapolated linearly from the pressure below the front (i.e., continuous phase), or is equal to the wetting phase pressure plus entry pressure (discontinuous phase). In the first case, the constant-potential criterion is conserved. However, local and average capillary pressures can become negative and this is a nonphysical result. In the second case, the constant-potential criterion is violated, but now local and average capillary pressures are physically acceptable as they do not become negative.

Acknowledgments The authors would like to thank Jan Martin Nordbotten for his advice regarding the centroid-corrected phase-average pressure. Rainer Helmig, Michael Celia, and Helge Dahle are gratefully acknowledged for many fruitful discussions. The first author would like to thank the Utrecht University master program System Earth Modeling for providing a scholarship to visit Stuttgart University. Authors are members of the International Research Training Group NUPUS, financed by the German Research Foundation (DFG) and the Netherlands Organisation for Scientific Research (NWO), and thank DFG (GRK 1398) and NWO (DN 81-754) for their support.

Open Access This article is distributed under the terms of the Creative Commons Attribution Noncommercial License which permits any noncommercial use, distribution, and reproduction in any medium, provided the original author(s) and source are credited. 


\section{Appendix: Determination of the Derivatives of the Intrinsic Phase-Volume Average Pressure and Position Vector}

In this section, we show the derivation of the derivative of the intrinsic phase-volume average pressure.

First, the definition of the intrinsic phase-volume averaged pressure (Eq. 2.1) is inserted into the derivative, assuming constant porosity:

$$
\nabla\left\langle P_{\alpha}\right\rangle^{i}=\nabla\left(\frac{1}{\int_{V} S_{\alpha} \mathrm{d} V} \int_{V} S_{\alpha} P_{\alpha} \mathrm{d} V\right)=\nabla\left(\frac{1}{\left\langle S_{\alpha}\right\rangle V} \int_{V} S_{\alpha} P_{\alpha} \mathrm{d} V\right) .
$$

By applying the product rule to Eq. A.1, we get:

$$
\nabla\left\langle P_{\alpha}\right\rangle^{i}=-\frac{\nabla\left\langle S_{\alpha}\right\rangle}{\left\langle S_{\alpha}\right\rangle^{2} V} \int_{V} S_{\alpha} P_{\alpha} \mathrm{d} V+\frac{1}{\left\langle S_{\alpha}\right\rangle V} \nabla\left(\int_{V} S_{\alpha} P_{\alpha} \mathrm{d} V\right) .
$$

The averaging theorem of Slattery (1967) states that:

$$
\nabla\langle\omega\rangle=\nabla\left\langle\frac{1}{V} \int_{V} \omega \mathrm{d} V\right\rangle=\frac{1}{V} \int_{\partial V} \mathbf{n} \omega \mathrm{d} V
$$

where $\omega$ denotes a function, $V$ is the averaging volume, $\mathbf{n}$ is the unit normal vector along the boundary of volume $\mathrm{V}$, denoted by $\partial V$.

Thus, the derivative of an average function $\omega$ can be expressed in terms of a surface integral. Using this theorem, Eq. A. 2 becomes:

$$
\nabla\left\langle P_{\alpha}\right\rangle^{i}=-\frac{\int_{\partial V} \mathbf{n} S_{\alpha} \mathrm{d} A}{\left\langle S_{\alpha}\right\rangle^{2} V^{2}} \int_{V} S_{\alpha} P_{\alpha} \mathrm{d} V+\frac{1}{\left\langle S_{\alpha}\right\rangle V} \int_{\partial V} \mathbf{n} S_{\alpha} P_{\alpha} \mathrm{d} A .
$$

The first term can be rewritten by applying the definition of the intrinsic phase-volume average pressure (Eq. 2.1):

$$
\nabla\left\langle P_{\alpha}\right\rangle^{i}=\frac{1}{\left\langle S_{\alpha}\right\rangle V}\left[-\left\langle P_{\alpha}\right\rangle^{i} \int_{\partial V} \mathbf{n} S_{\alpha} \mathrm{d} A+\int_{\partial V} \mathbf{n} S_{\alpha} P_{\alpha} \mathrm{d} A\right] .
$$

A similar approach can be followed in the derivation of the derivative of the intrinsic phase-volume average position vector $z$, resulting in:

$$
\nabla \cdot\left\langle z_{\alpha}\right\rangle^{i}=\frac{1}{\left\langle S_{\alpha}\right\rangle V}\left[-\left\langle z_{\alpha}\right\rangle^{i} \cdot \int_{\partial V} n \cdot S_{\alpha} \mathrm{d} A+\int_{\partial V} n \cdot S_{\alpha} z \mathrm{~d} A\right] .
$$

For the particular situation of a 1-D vertical domain and flow from the bottom of the domain to the top, Eq. A.5 and A.6 become (Nordbotten, personal communication):

$$
\begin{aligned}
\frac{\partial}{\partial z}\left\langle P_{\alpha}\right\rangle^{i} & \equiv \frac{1}{\left\langle S_{\alpha}\right\rangle H}\left[-\left\langle P_{\alpha}\right\rangle^{i}\left(S_{\alpha}^{\text {top }}-S_{\alpha}^{\text {bottom }}\right)+\left(S_{\alpha}^{\text {top }} P_{\alpha}^{\text {top }}-S_{\alpha}^{\text {bottom }} P_{\alpha}^{\text {bottom }}\right)\right] \\
\frac{\partial}{\partial z}\left\langle z_{\alpha}\right\rangle^{i} & \equiv \frac{1}{\left\langle S_{\alpha}\right\rangle H}\left[-\left\langle z_{\alpha}\right\rangle^{i}\left(S_{\alpha}^{\text {top }}-S_{\alpha}^{\text {bottom }}\right)+\left(S_{\alpha}^{\text {top }} z^{\text {top }}-S_{\alpha}^{\text {bottom }} z^{\text {bottom }}\right)\right],
\end{aligned}
$$


where $H$ is the height of the modeling domain. The superscripts "top" and "bottom" refer to the value of the variable $\left(S_{\alpha}, P_{\alpha}\right.$, and $\left.z\right)$ at the top and the bottom of the domain, respectively.

\section{References}

Ataie-Ashtiani, B., Hassanizadeh, S.M., Oostrom, M., Celia, M.A., White, M.D.: Effective parameters for two-phase flow in a porous medium with periodic heterogeneities. J. Contam. Hydrol. 49, 87-109 (2001)

Ataie-Ashtiani, B., Hassanizadeh, S.M., Celia, M.A.: Effects of heterogeneities on capillary pressuresaturation-relative permeability relationships. J. Contam. Hydrol. 56, 175-192 (2002)

Bachmat, Y., Bear, J.: Macroscopic modeling of transport in porous media, Part 1: The continuum approach. Transp. Porous Media 1(3), 213-240 (1986)

Bear, J.: Dynamics of Fluids in Porous Media, 2 edn, pp. 761. Dover Publ., New York (1988)

Bear, J., Bachmat, Y.: Macroscopic modeling of transport in porous media, Part 2: Applications to mass, momentum and energy transport. Transp. Porous Media 1(3), 241-270 (1986)

Dahle, H.K., Celia, M.A.: A dynamic network model for two-phase immiscible flow. Comput. Geosci. 3, $1-22(1999)$

Dahle, H.K., Celia, M.A., Hassanizadeh, S.M.: Bundle-of-tubes model for calculating dynamic effects in the capillary pressure-saturation relationship. Transp. Porous Media 58(1-2), 5-22 (2005)

Das, D.B., Hassanizadeh, S.M., Rotter, B.E., Ataie-Ashtiani, B.: A numerical study of micro-heterogeneity effects on upscaled properties of two-phase flow in porous media. Transp. Porous Media 56, 329-350 (2004)

Gielen, T.W.J.: Dynamic effect in two-phase flow in porous media: a pore-scale network approach. Ph.D.Thesis, Utrecht University, p. 175 (2007)

Gielen, T.W.J., Hassanizadeh, S.M., Celia, M.A., Dahle, H.K., Leijnse, A.: A pore-scale network approach to investigate dynamic effects in multiphase flow. Proc. CMWR XV Conference, University of North Carolina (2004)

Gielen, T.W.J., Hassanizadeh, S.M., Nordhaug, H.F., Leijnse, A: Dynamic effects in multiphase flow: a porescale network approach. In: Das, D.B., Hassanizadeh, S.M. Upscaling Multiphase Flow on Porous Media: From Pore to Core and Beyond, Springer, Dordrecht (2005)

Gray, W.G.: A derivation of the equations for multi-phase transport. Chem. Eng. Sci. 30, 229-233 (1975)

Gray, W.G., Miller, C.T.: Examination of Darcy's law for flow in porous media with variable porosity. Environ. Sci. Technol. 38, 5895-5901 (2004)

Gray, W.G., Miller, C.T.: Consistent thermodynamic formulations for multiscale hydrologic systems: Fluid pressures. Water Resour. Res. 43, W09408 (2007). doi:10.1029/2006WR005811

Gray, W.G., O'Neill, K.: On the general equations for flow in porous media and their reduction to Darcy's law. Water Resour. Res. 12(2), 148-154 (1976)

Hassanizadeh, S.M., Gray, W.G.: General conservation equations for multi-phase systems: 1-Averaging procedure. Adv. Water Resour. 2, 131-144 (1979)

Helmig, R.: Multiphase Flow and Transport Processes in the Subsurface: A contribution to the modeling of hydrosystems. Springer, Berlin, ISBN:3-540-62703-0 (1997)

Manthey, S., Hassanizadeh, S.M., Helmig, R.: Macro-scale dynamic effects in homogeneous and heterogeneous porous media. Transp. Porous Media 58, 121-145 (2005)

Neumann, S.P.: Theoretical derivation of Darcy's law. Acta Mech. 25, 153-170 (1977)

Nordbotten, J.M., Celia, M.A., Dahle, H.K., Hassanizadeh, S.M.: Interpretation of macroscale variables in Darcy's law. Water Resour. Res. 43, W08430 (2007). doi:10.1029/2006WR005018

Nordbotten, J.M., Celia, M.A., Dahle, H.K., Hassanizadeh, S.M.: On the definition of macro-scale pressure for multi-phase flow in porous media. Water Resour. Res. 44, W06502 (2008). doi:10.1029/2006WR005715

Quintard, M., Whitaker, S.: Transport in ordered and disordered porous media I: the cellular average and the use of weighting functions. Transp. Porous Media 14, 163-177 (1994a)

Quintard, M., Whitaker, S.: Transport in ordered and disordered porous media II: generalized volume averaging. Transp. Porous Media 14, 179-206 (1994b)

Slattery, J.C.: Flow of viscoelastic fluids through porous media. AIChE J. 13, 1066-1071 (1967)

Whitaker, S.: Simultaneous heat, mass, and momentum transfer in porous media: a theory of drying. Adv. Heat Transf. 13 (1997)

White, M.D., Oostrom, M.: STOMP, Subsurface Transport Over Multiple Phases. User's Guide. Pacific Northwest National Laboratory Report PNNL-11218 (1997) 